\title{
Research Paper: Effect of Cognitive Rehabilitation on Executive Functions in Adoles- cent Survivors of Leukemia: A Randomized and Controlled Clinical Trial
}

\author{
*Omid Amani ${ }^{1}$, Mohammad Ali Mazaheri ${ }^{1}$, Vahid Nejati ${ }^{1}$, Bibi Shahin Shamsian ${ }^{2}$
}

1. Department of Psychology, Faculty of Education and Psychology, Shahid Beheshti University, Tehran, Iran.

2. Department of Applied Cell Sciences, School of Advanced Technologies in Medicine, Shahid Beheshti University of Medical Sciences, Tehran, Iran.

ditation: Amani O, Mazaheri MA, Nejati V, Shamsian BS. [Effect of Cognitive Rehabilitation on Executive Functions in Adolescent Survivors of Leukemia: A Randomized and Controlled Clinical Trial (Persian)]. Archives of Rehabilitation. 2017; 18(1):73-82. https://doi.org/10.21859/jrehab-180173

: https://doi.org/10.21859/jrehab-180173

Received: 13 Oct. 2016

Accepted: 11 Feb. 2017

Keywords:

Cognitive rehabilitation, Executive functions, Hope, leukemia

\section{A B STRACT}

Objective One of the phenomena that have been observed among survivors of childhood cancers like leukemia is the late side effects of the applied treatments, such as chemotherapy, on executive cognitive functions. These problems lead to various complaints such as experiencing dullness while performing activities, encountering difficulties in conducting various tasks simultaneously, and having no interest in laying out plans. These issues will endanger and negate the advantage of any increase in survival rate. Therefore, the aim of this clinical trial is to evaluate the efficiency of using a series of cognitive rehabilitation exercises in improving executive functions on the level of hope in adolescents who survived leukemia.

Methods \& Materials This research is a quasi-experimental study with pretest, posttest, and follow-up stages. The study population consisted of all the adolescents between the ages of 12 to 18 years, who survived leukemia, in Tehran city. Purposive sampling method was used. Based on the medical records and inclusion and exclusion criteria, 30 patients, who had been referred to the section of blood diseases at the Mofid hospital in the year 2013, were selected and accidentally classified into intervention (15) and wait list (15) groups. The participants of the intervention group received treatment offered as part of the cognitive rehabilitation program over a period of 12 sessions. The research data was collected using the Wechsler Intelligence Scale, Achenbach Behavior Scale, and the Miller Hope Test. These data were analyzed with the help of descriptive statistics (mean and standard deviation) and inferential statistics (multivariate analysis of covariance).

Results The mean treatment duration was found to be 23.27 months in the intervention group and 27.00 months in the waiting list group. The mean treatment completion time of the intervention group is obtained as 25.60 , and in the waiting list group as 30.67. The findings of the Miller hope questionnaire revealed that executive functions witnessed a significant positive effect due to cognitive rehabilitation, in adolescents who survived leukemia $(P<0.05)$. This effect remained stable, as shown in a follow-up of one month after the intervention $(P<0.05)$.

Conclusion The results of this study have shown that the effect of cognitive rehabilitation on executive functions reaps significant dividends with regard to the promotion of hope in adolescents who survived leukemia and have been under the chemotherapy. The promotion of hope in participants belonging to the intervention group was more than in the participants of the wait list group. Given the fact that, today, one of the main goals of treatment of chronic diseases such as cancer is to enhance the quality of mental health of the surviving patients, the use of inexpensive and accessible programs like cognitive rehabilitation would be very effective. Consequently, healthcare professionals could attempt to reduce the side effects caused by chemotherapy and radiation, in addition to improving the cognitive issues of affected individuals, by placing emphasis on hope among the concerned patients. Therefore, it is recommended to conduct further research by considering the results of this work in order to develop effective cognitive rehabilitation interventions, thereby, enhancing the coping strategies, quality of life, and mental health of patients who are afflicted with cancer and those who survived cancer. Cognitive rehabilitation services should also be considered for post-treatment follow-up projects.

\section{* Corresponding Author:}

Omid Amani, MSc.

Address: Department of Psychology, Faculty of Education and Psychology, Shahid Beheshti University, Tehran, Iran.

Tel: +98 (921) 2631959

E-Mail: o.amani69@yahoo.com 


\title{
تأثير توانبخشى شناختى كاركردهاى اجرايى بر افزايش ميزان اميدوارى نوجوانان نجاتيافته از

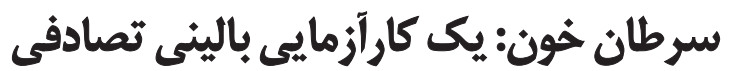

\author{
"اميد امانى'، محمدعلى مظاهرى'، وحيد نجاتى '، بىبى شهين شمسيان' \\ ا - كروه روانشناسى، دانشكده علوم تربيتى وروانشناسى، دانشكاه شهيد بهشتى، تهران، ايران.

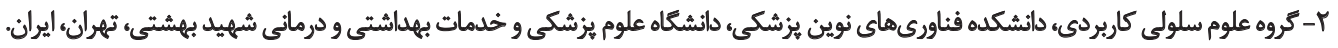

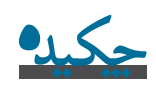

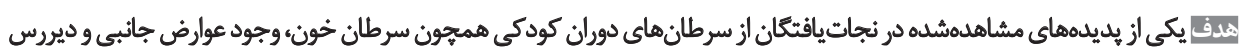

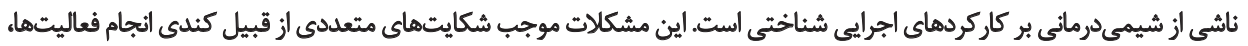

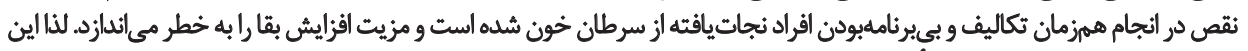

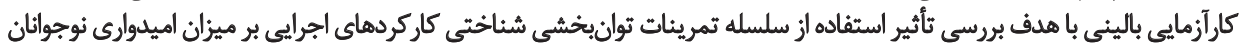

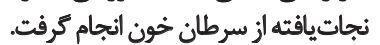

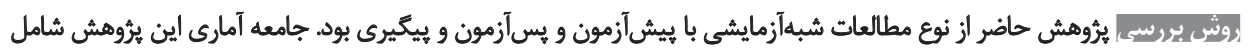

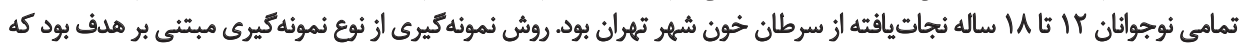

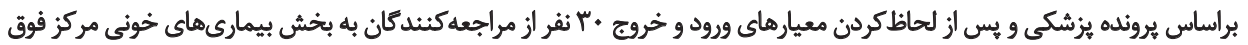

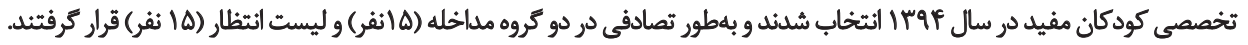

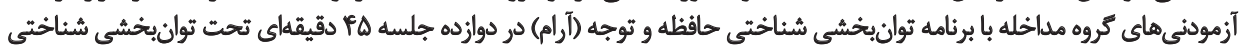

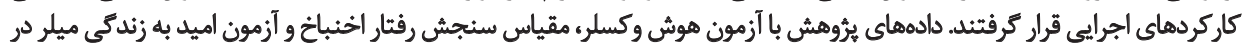

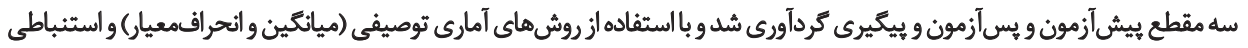

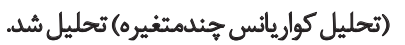

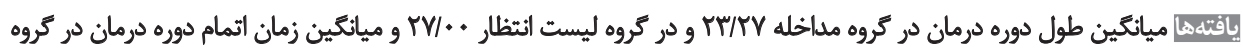

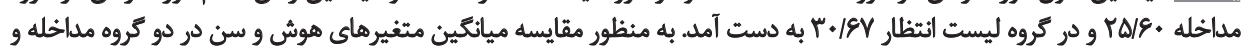

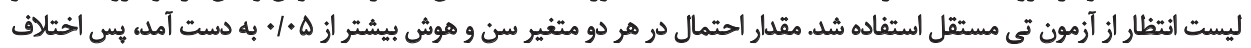

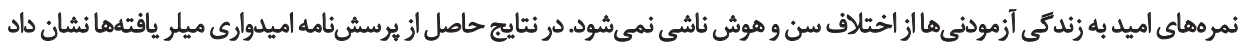

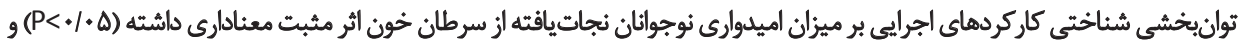

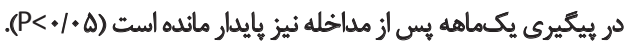

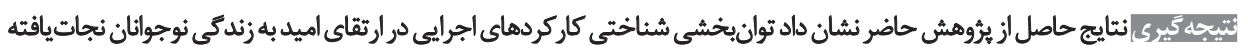

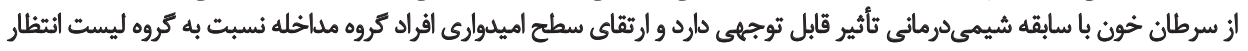

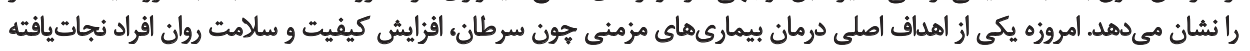

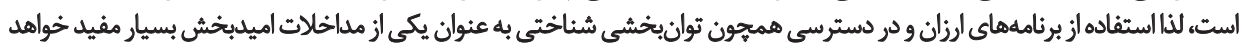

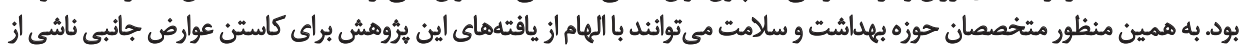

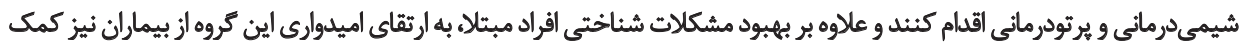

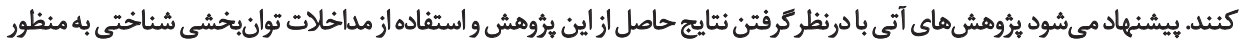

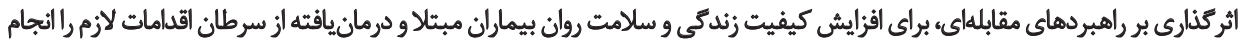

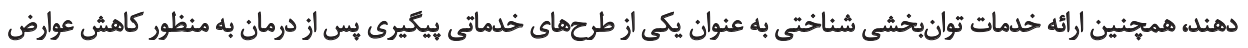

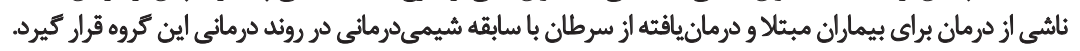

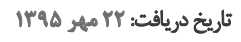

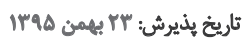

كليدواثهها:

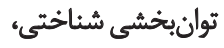

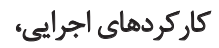
اميد، سرطان خون أجرايى 
توانايىهاى ههم همجون توجه، حافظه فعال، تصميمكيرى، مقلمه

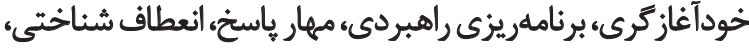

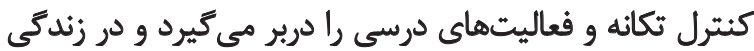

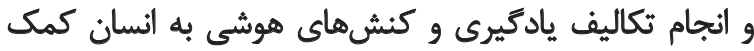

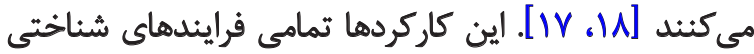

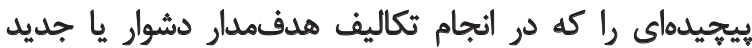

ضرورى هستند در خود جاى مي دهام تكاليف [19]

يرؤششهاى متعددى به بررسى اثرات شيمىدرمانى در

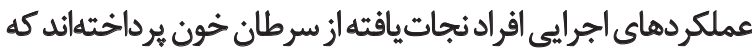

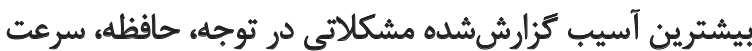

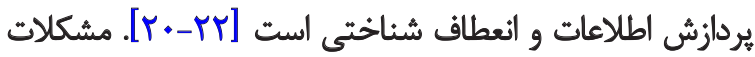

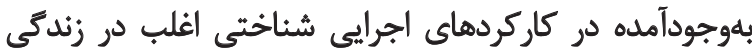

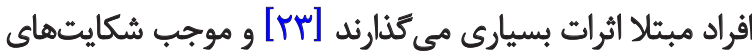

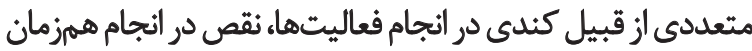

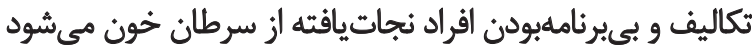

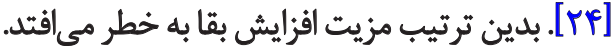

يكى از راههاى بهبود مشكلات عصبشناختى ايجادشده در الئي

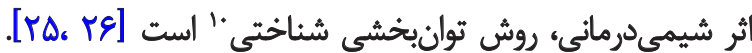

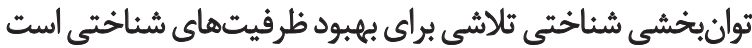

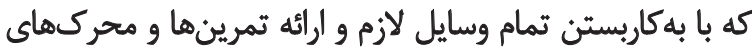

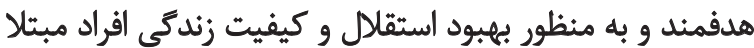

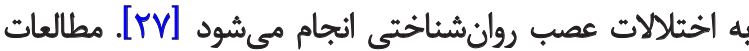

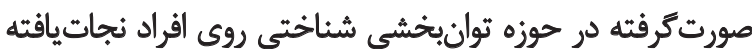

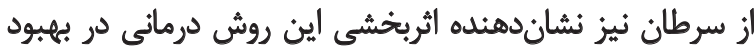

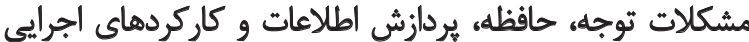

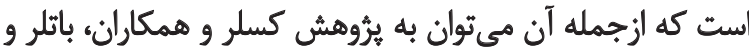

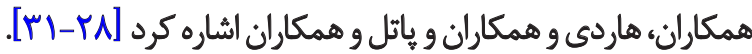

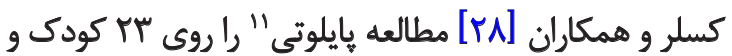

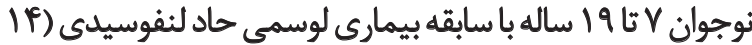

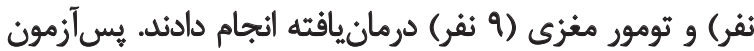

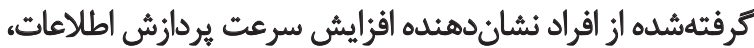

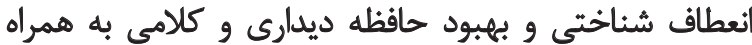

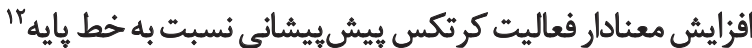

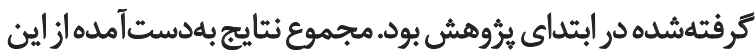

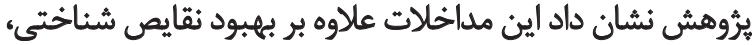

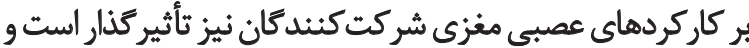
امكان افزرايش فعاليتهاي عصبى را فراهم مي كندئ

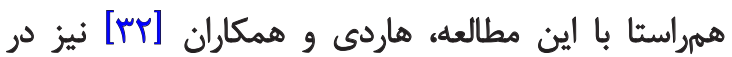

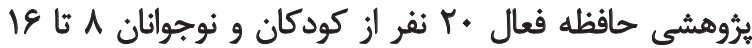

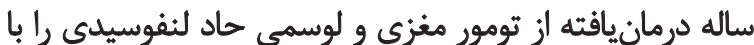

سرطان' به عنوان يك بيمارى مزمن بعد از تصادفات و ومان

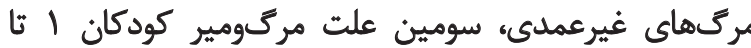

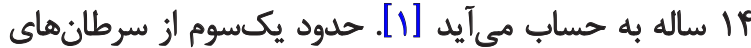

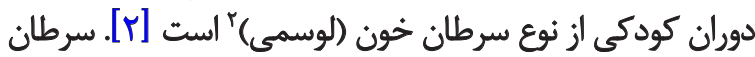

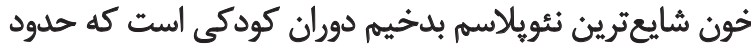

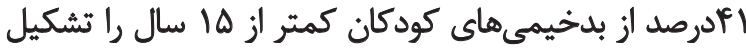

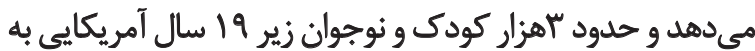

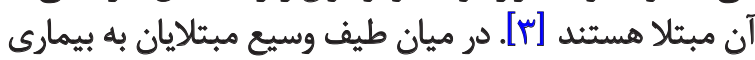

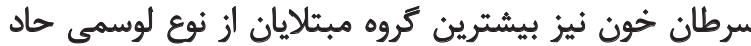

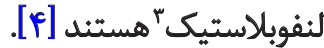

شيوع لوسمى حاد لنفوبلاستيك در يسرها بيشتر از دختران

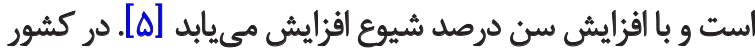

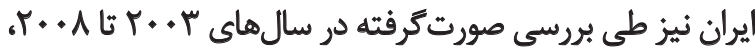

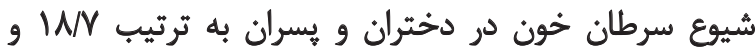

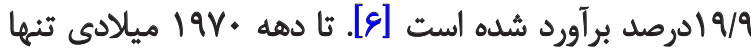

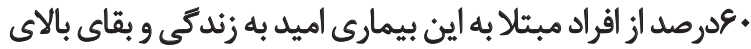

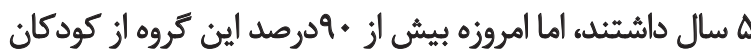

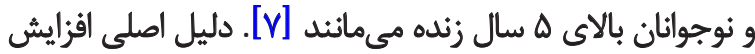

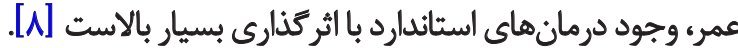
به موازات افزايش نجات يافتكان "از اين بيمارى، تمركز مداخلات

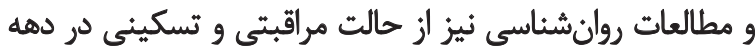
19V.

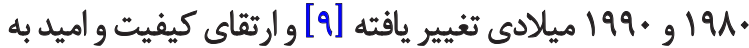

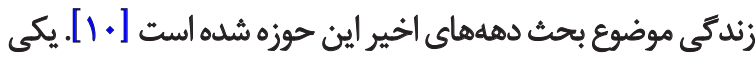

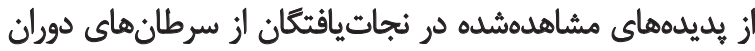

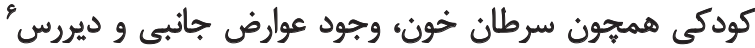

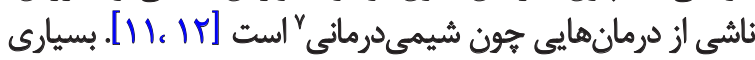

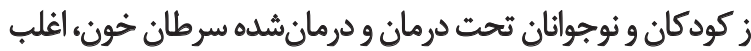

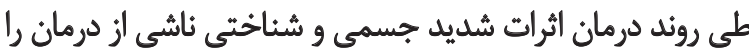

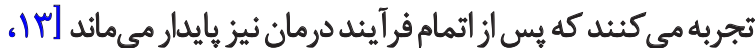

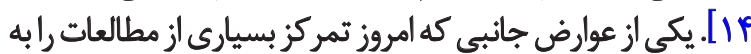

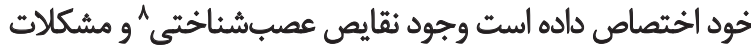

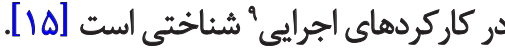

كاركردهاى اجرايى به سلسلهمراتب بالاتر و خودتنظيمكر

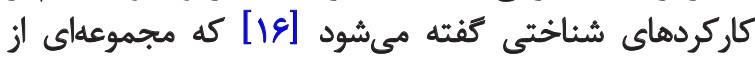

1. Cancer

2. Leukemia

3. Acute lymphoblastic Leukemia (ALL)

4. Survivors

5. Side effect

6. Late effect

7. Chemotherapy

8. Neuropsychologica

9. Executive function 


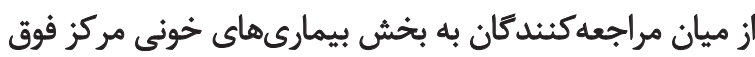

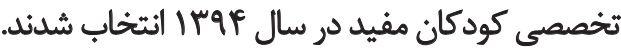

به منظور تردآورى دادهها، ابتدا روش انجام مطالعه به

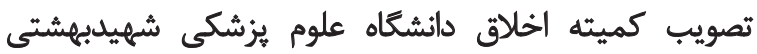
به شماره ثبت و در مركز بينالمللى ثبث كارآزمايىهاى بالينى ايران با كد باري

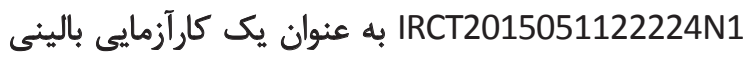
ثبت شد. در كام بعدى محققان با مراجعه به بيمارستان كودكان بان باني

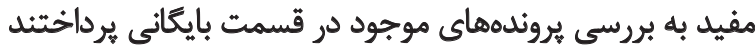

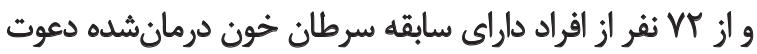

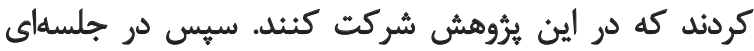

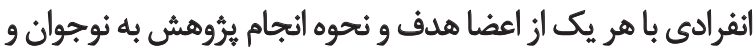

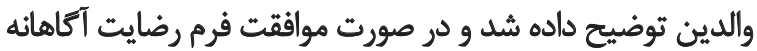

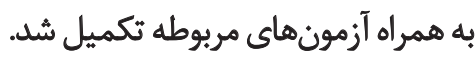

در كام بعد محققان به بررسى دادههاى بهدست آمده از مرحله

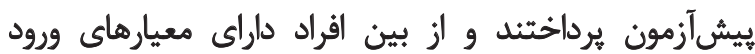

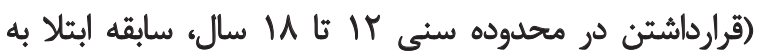

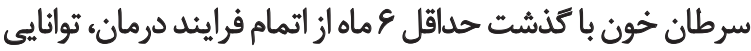

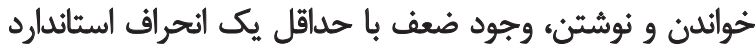

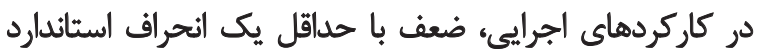

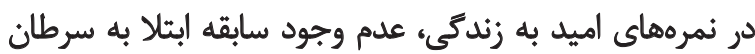

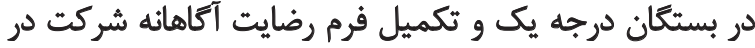

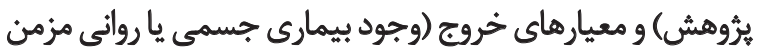

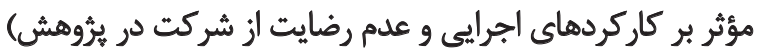

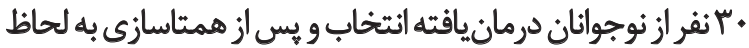

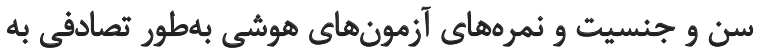
دو كروه مداخله (ها نفر) و ليست انتظار (ها نفر نفر) تقسيم شدند.

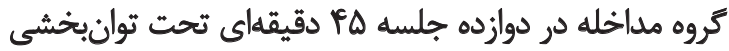

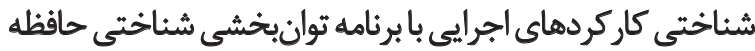

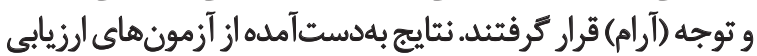

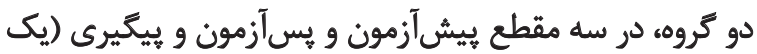

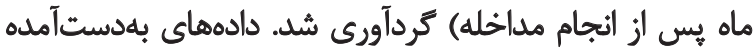

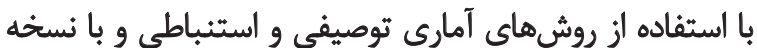

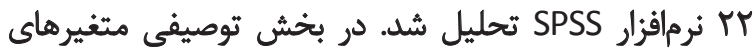

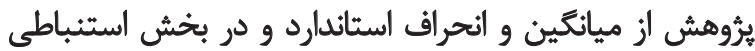

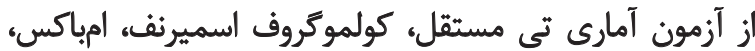

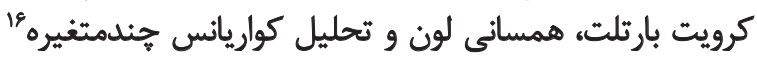

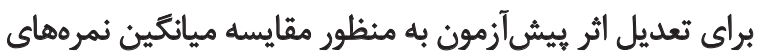

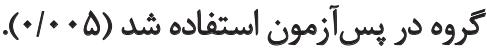

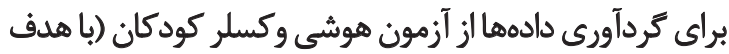

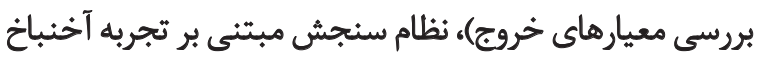

16. MANCOVA
استفاده از يك برنامه توانبخشى شناختى كامييوترى خانتكى به

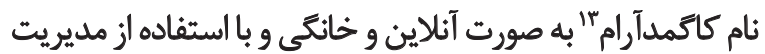

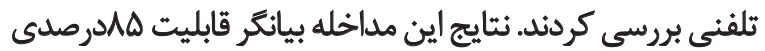

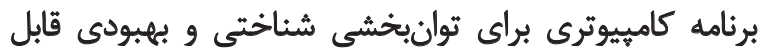

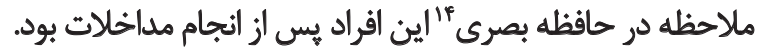

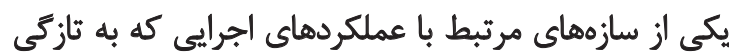

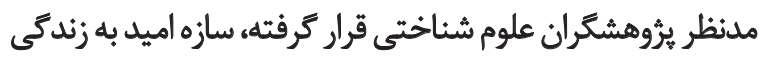

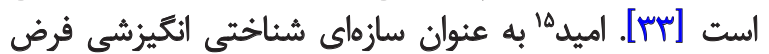

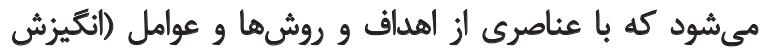

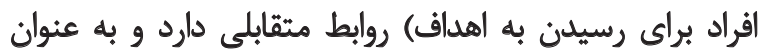

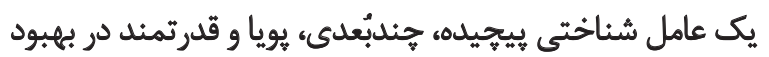

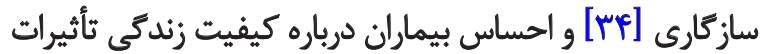

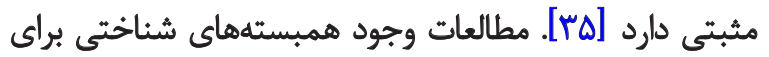

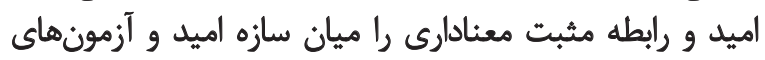

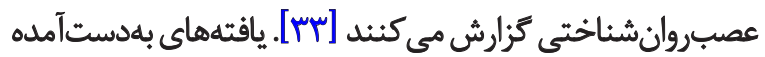

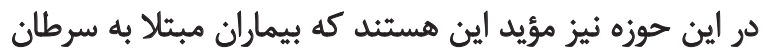

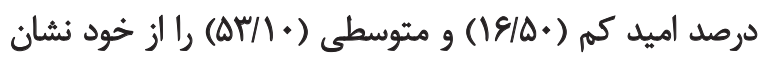

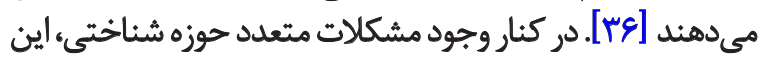

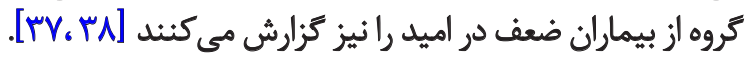
با نتاهي موشكافانه به مطالعات انجامشده در زمينه كاركردهاي

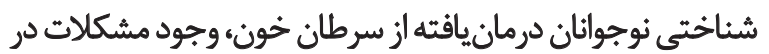

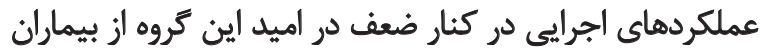

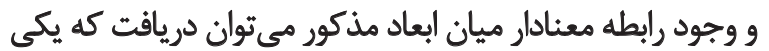

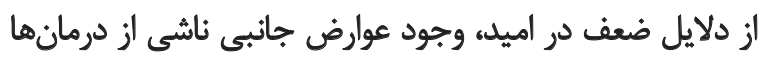

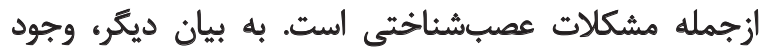

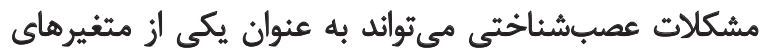

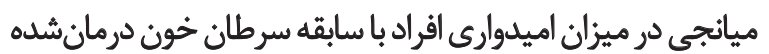

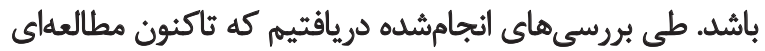

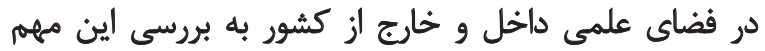

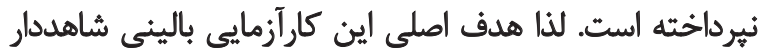

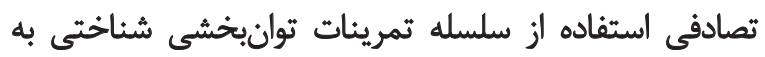
منظور تقويت كاركردهاى اجرايي و مطالعه اثرات آن بآن بر ميزان آنان

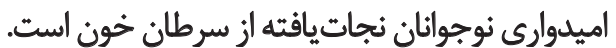

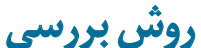

يثروهش حاضر از نوع مطالعات كارآزمايى بالينى تصادفى با ناريا

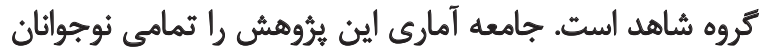

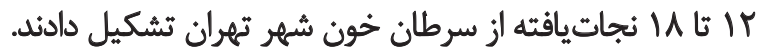

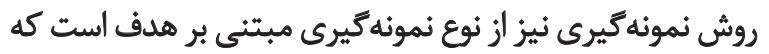

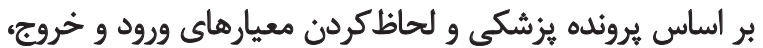

\section{Cogmed RM}

14 . Visual working memory

15. Hope 
هنجاريابى اين آزمون در ايران ضرايب همسانى درونى مقياسهاو

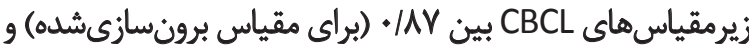

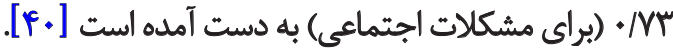

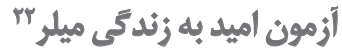

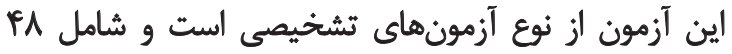

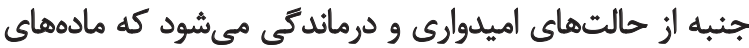

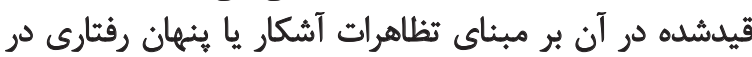

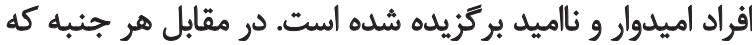

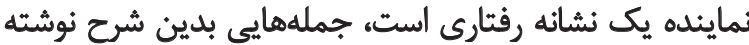

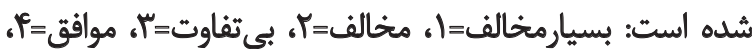

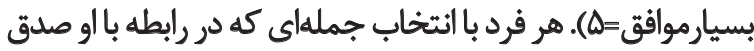

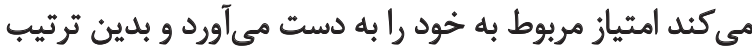

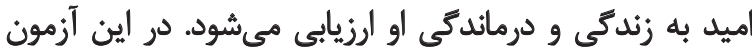

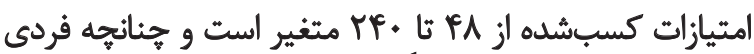

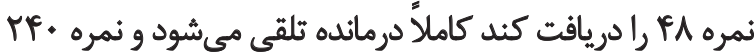
نيز حداكثر اميدوارى رانشان مي دهند. نماندان

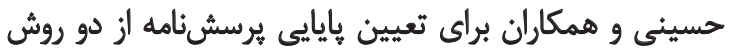

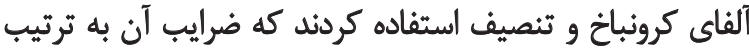

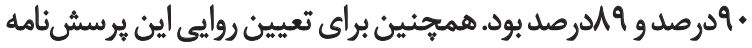

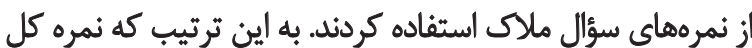

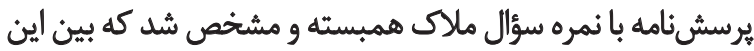

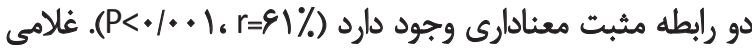

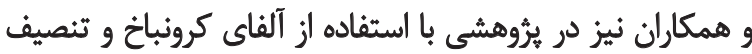

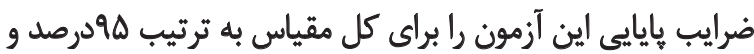
Fif

$$
\text { برئامه توانبخشى شناختى ثوجه و حافظله (آرام) }
$$

تقويت عملكردهاى اجرايي با استفاده از برنامه توانبخشى تواري

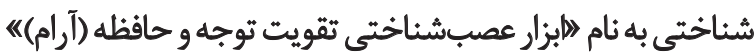

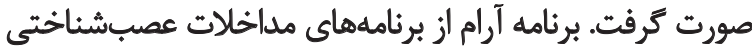

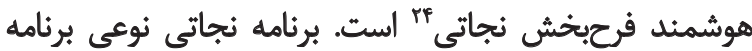

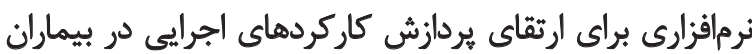

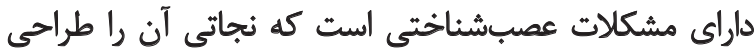

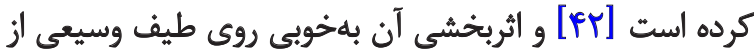
مبتلايان به اختلالات شناختى بررسى شده آند است.

اين برنامه شامل گروهى از تكاليف سلسلهمراتبى سازماندهى

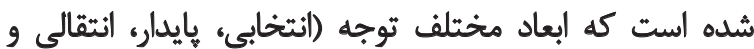

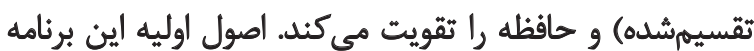

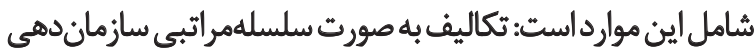

\section{Miller Hopeful Inventory (MHI)}

23. Attentive Rehabilitation of Attention and Memory (ARAM)

24. NEuroconitive Joyful Attentive Training Intervention (NEATI)
(بSEBA)

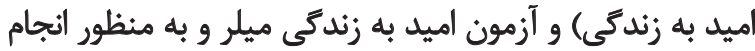

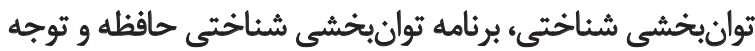

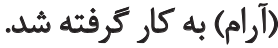

$$
\text { آزمون هوش وكسلر كودكان " }
$$

مقياس تجديدنظرشده هوش كودكان شامل آزمونهاي هوشى

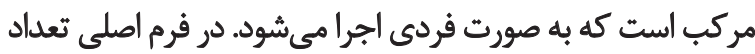

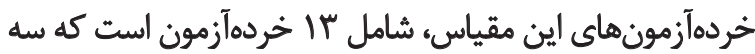

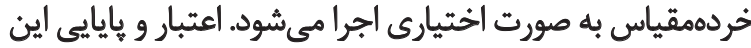

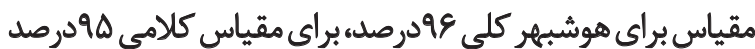

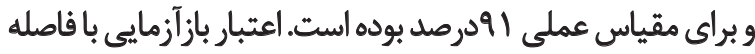

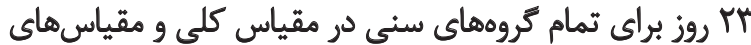

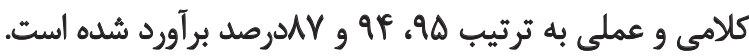

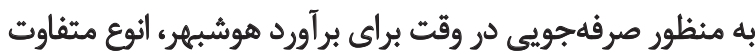
فرمهاى كوتامدت تدوين شوند

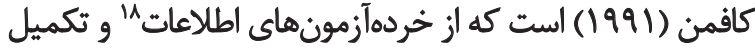

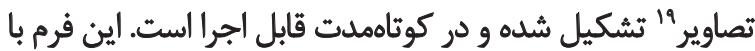

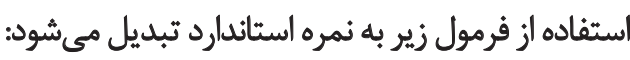
(I+PC)+FT اين فرم كوتاه با مقياس كلى ضريب همبستگى بين عمدادرصد

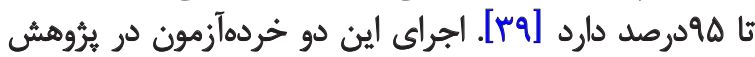
حاضر بلهور تقريبى • ال دقيقه طول كشيد.

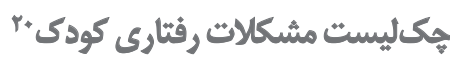

در اين ثروهش براى سنجش مشكلات رفتّارى ثأثيركذار بر

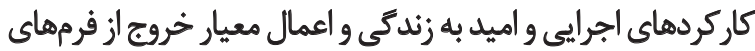

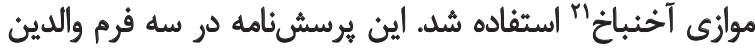

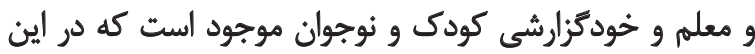

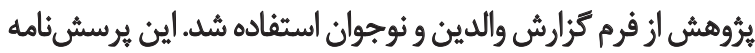

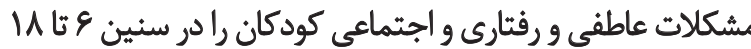

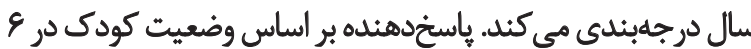

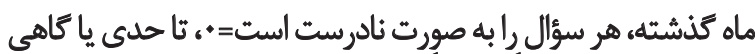

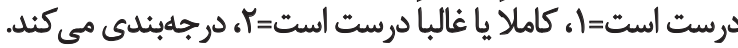
سؤال هاى سندرمى فرمهاى آخنباخ در نيمرخ سندرمها شامل

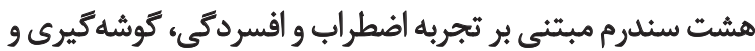

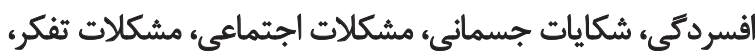

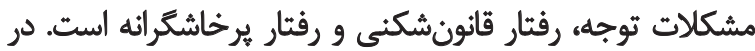

17. Wechsler Intelligence Scale for Children (WISC-III)

18. Information

19. Picture completion

20. Child Behavior Checklist (CBCL)

21. Achenbach 


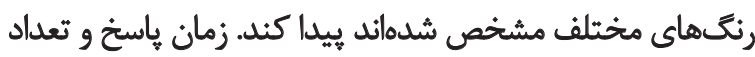
ياسخ صحيح به عنوان خروجى تكليف ثبت مئش مشود.

\section{تكليف انتقال و مهار}

در اين تكليف فرد بر اساس يك قانون متغير مجموعه محرك انها

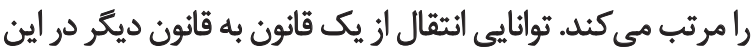

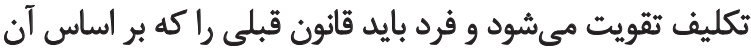

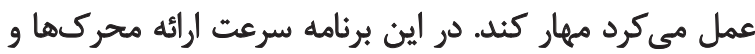

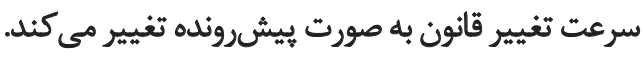

يافتهها

در كارآزمايع بالينى حاضر اطلاعات مربوط به ·ـ نو نوجوان

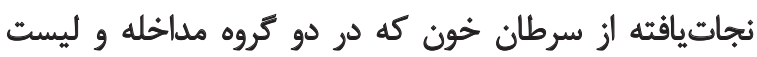

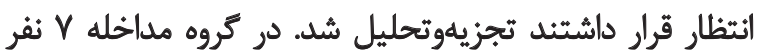

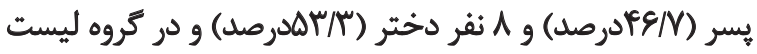

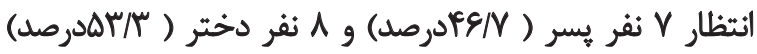

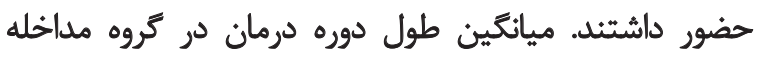
دور دr/TY

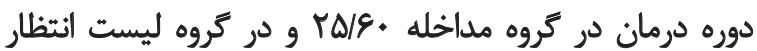

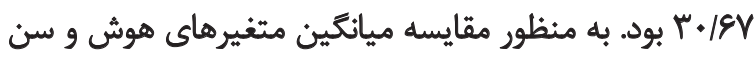

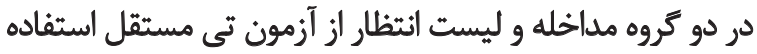

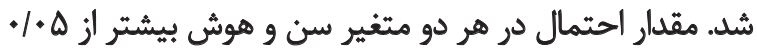

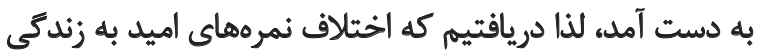

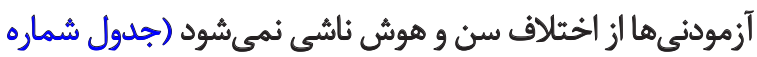

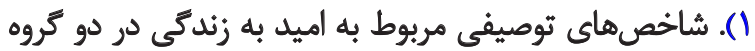

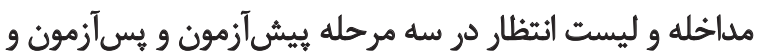
ييكيرى در جدول شماره بار ارائه شده است

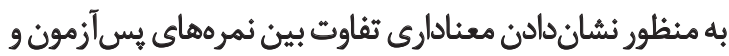

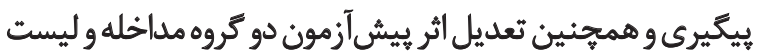

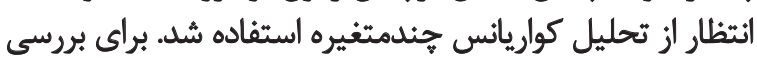

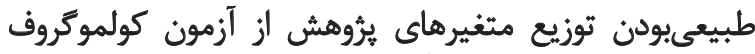

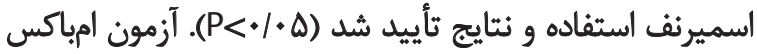

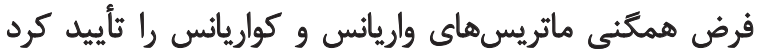

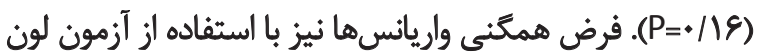

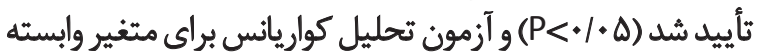

شداند و مبتنى بر ياسخ كاربر طي جلسات سخت بـر مي بشوند؛

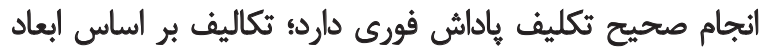

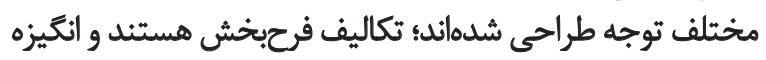

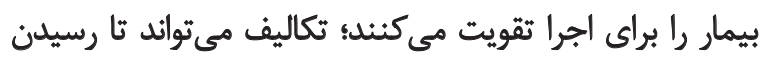

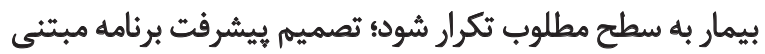

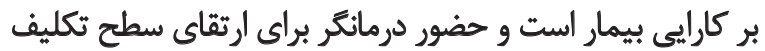
نياز است اجزايى برنامه توانبخشى شناختى شامل موارد زير است:

تكليف آمادتى

اين تكليف به صورتى است كه فرد توجه خود را براى يك محرى

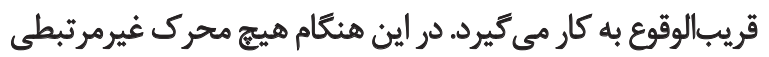

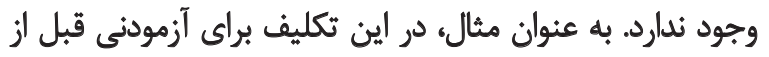

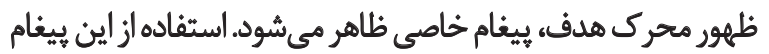

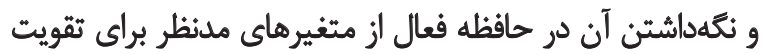

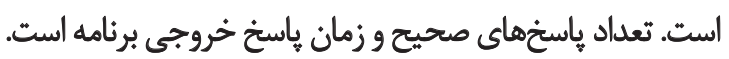

\section{تكليف جستوجو و انتخاب}

در اين تكليف يك محرك به عنوان محرك هدف براي آزمودنى

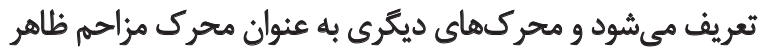

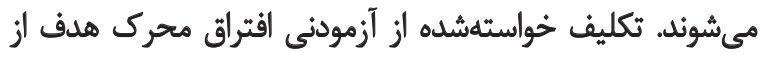

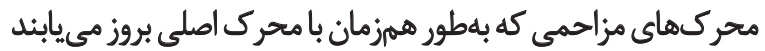

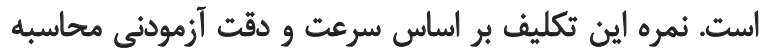

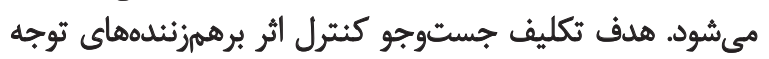

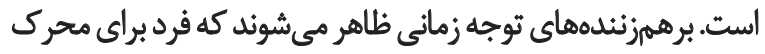

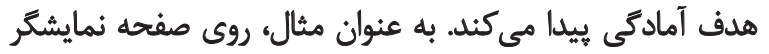

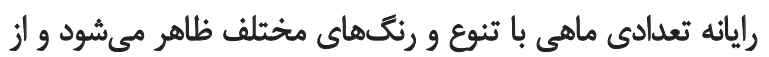

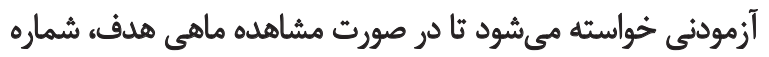

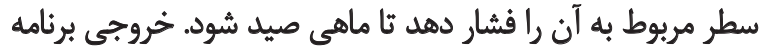

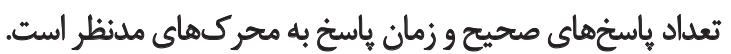

تكليف نتَّهدارى

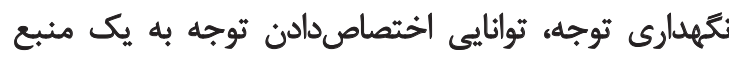

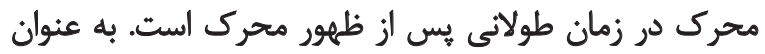

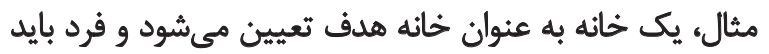

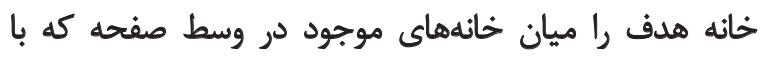

جدول 1. مقايسه سن و هوش آزمودنىها در دو تروه مداخله و ليست انتظار

\begin{tabular}{|c|c|c|c|c|c|}
\hline مقدار احتمال & آماره تى & انحراف معيار & ميانكين & كروهها & متغيرها \\
\hline \multirow{2}{*}{ 年 } & \multirow{2}{*}{$V / \pi T$} & $1 / 491$ & $1 r / q r$ & مداخله & \multirow[b]{2}{*}{ سن } \\
\hline & &.$/ 941$ & $15 / 19$ & ليست انتظار & \\
\hline . / & • & $\begin{array}{l}P N \cdot F \\
F / A \circ F\end{array}$ & $\begin{array}{l}\text { धा.. } \\
\text { धारा }\end{array}$ & لميست اتخظار & هوش \\
\hline
\end{tabular}


جدول Y. شاخصهاي آمارى اميد به زندكى در كروه مداخله و ليست انتظار

\begin{tabular}{|c|c|c|c|c|c|}
\hline \multicolumn{2}{|c|}{ تروه ليست انثظار } & \multicolumn{2}{|c|}{ مروه مداخله } & \multirow{2}{*}{ موقعيت } & \multirow{2}{*}{ متغير } \\
\hline انحراف استاندارد & مياتكين & انحراف استاندارد & مياتكين & & \\
\hline$|F / \Delta|$ & $10 \cdot / \Delta t$ & WOA & $\mid F q / T H$ & ييش أزمون & \\
\hline$I V / \Delta V$ & IPV/ge & 9/99 & $109 / \%$. & يسآزمون & اميد به زندكى \\
\hline$i f / N F$ & IFNT.. & $10 / 1$ & $\mid E T / \Delta T$ & ييكيرى & \\
\hline
\end{tabular}

توانبخننى

جدول ؟. نتايج آزمون تحليل كواريانس جندمتغيره بررسى تأثير توانبخشى شناختى كاركردهاى اجرايى بر اميد به زندكى در نوجوانان نجاتياقته از سرطان خون

\begin{tabular}{|c|c|c|c|c|c|c|c|}
\hline مجذور اتا & سطح معنادارى & 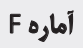 & ميانكين مجذورات & درجات أزادى & مجموع مجذوروات & \multicolumn{2}{|c|}{ شاحُص منابع تغييرات } \\
\hline.$/ 198$ & I/ YA & $\Delta / T \Delta A$ & QFV/TaY & $m, 1$ & $9 \% / / T 97$ & اميد به زندكىى & اثر كروه براى يس آزمون \\
\hline$. / \pi+8$ & سוּ/. & 8/994 & IATT/ATA & $r n, 1$ & IATE/ATA & اميد به زندكىى & اثر كروه براى بييكيرى \\
\hline
\end{tabular}

كاركردهاى اجرايي، نمرههاى اميد نيز ارتقا يافته باشد كه اين

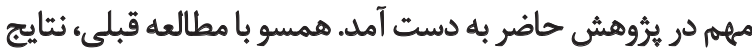

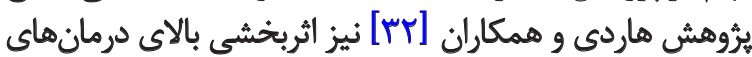

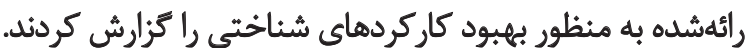

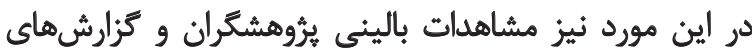

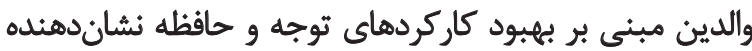

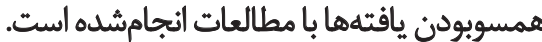

نتايج اين بثروهش را مى توان از دو موضع بررسى كرد؛ موضع

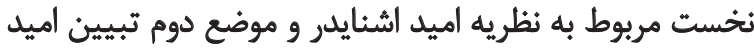

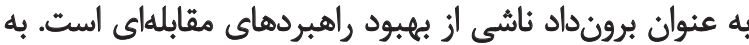

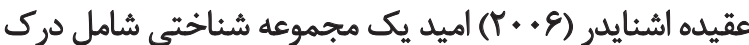

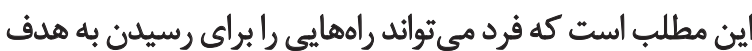

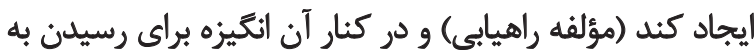

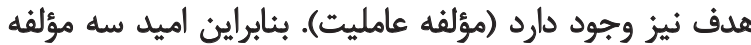

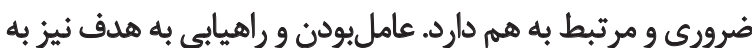

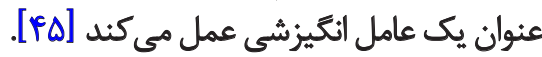
مطالعه نجاتى و همكاران [سب] نيز نشاندهنده همبستكئى

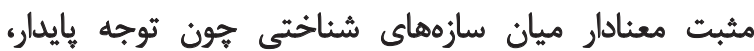

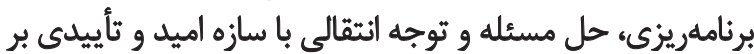

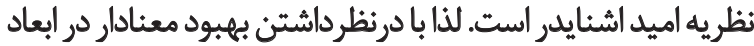

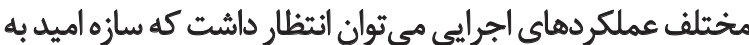

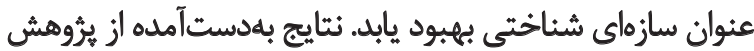

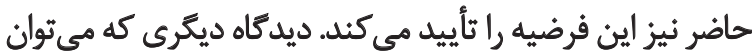

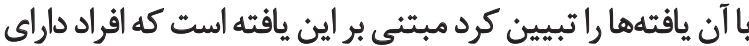

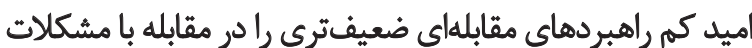

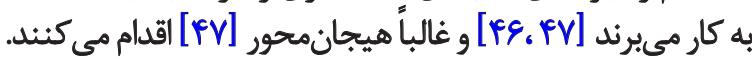

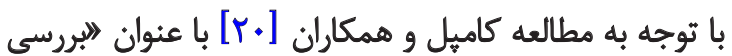

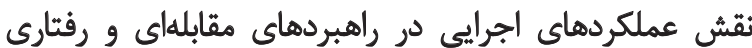

محاسبه شد كه نتايج آن در جدول شماره بَ آمده است.

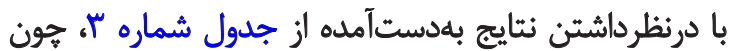

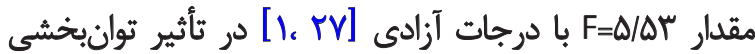

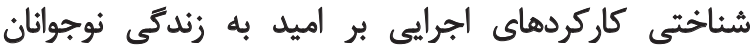

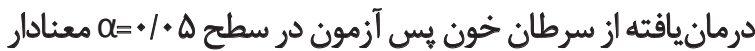

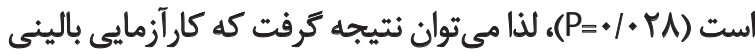

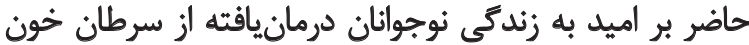

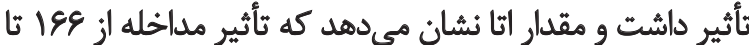

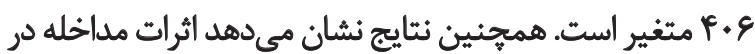

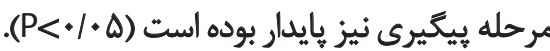

بحث

نتايج بروهش حاضر نشاندهنده تغييرات قابل ملاحظه

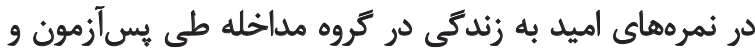

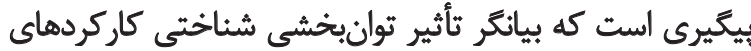

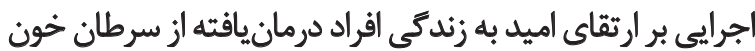

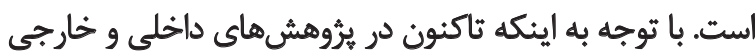

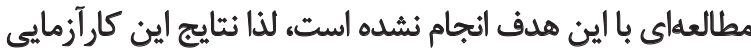
با يافتههاى مطالعات مرتبط ديخً بررسى شد.

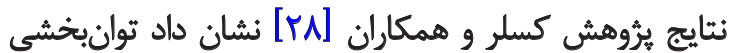

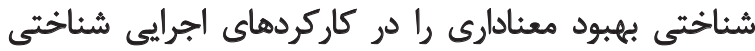

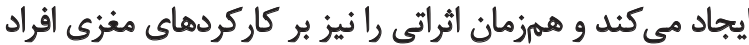

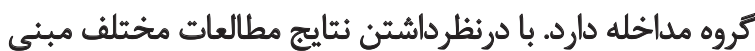

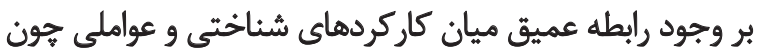

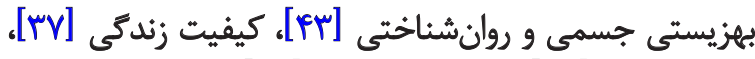

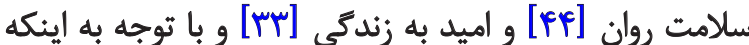

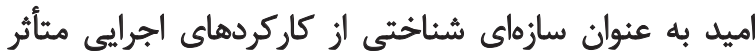

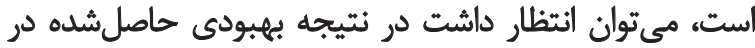




\section{References}

[1] Gatta G, Zigon G, Capocaccia R, Coebergh JW, Desandes E, Kaatsch P, et al. Survival of European children and young adults with cancer diagnosed 1995-2002. European Journal of Cancer. 2009; 45(6):992-1005. doi: 10.1016/j.ejca.2008.11.042

[2] Siegel R, Ward E, Brawley O, Jemal A. Cancer statistics, 2011. CA: A Cancer Journal for Clinicians. 2011; 61(4):212-36. doi: $10.3322 /$ caac.20121

[3] Hunger SP, Lu X, Devidas M, Camitta BM, Gaynon PS, Winick NJ, et al. Improved survival for children and adolescents with acute lymphoblastic leukemia between 1990 and 2005: A report from the children's oncology group. Journal of Clinical Oncology. 2012; 30(14):1663-69. doi: 10.1200/jco.2011.37.8018

[4] Linet MS, Dores GM, Kim CJ, Devesa SS, Morton LM. Epidemiology and hereditary aspects of acute leukemia. Neoplastic Diseases of the Blood. Philadelphia: Springer; 2013.

[5] Kliegman RM. Nelson textbook of pediatrics. Philadelphia: Springer; 2012

[6] Koohi F, Salehiniya H, Shamlou R, Eslami S, Ghojogh ZM, Kor $\mathrm{Y}$, et al. Leukemia in Iran: Epidemiology and morphology trends. Asian Pacific Journal of Cancer Prevention. 2014; 16(17):775963. PMID: 26625794

[7] Pui CH, Campana D, Pei D, Bowman WP, Sandlund JT, Kaste SC, et al. Treating childhood acute lymphoblastic leukemia without cranial irradiation. New England Journal of Medicine. 2009; 360(26):2730-41. doi: 10.1056/nejmoa0900386

[8] Smit A, Smit A. Experiences of gynaecological cancer and treatment of female survivors [MSc. thesis]. Palmerston North, New Zealand: Massey University; 2011.

[9] Cooper GM. The cancer book: A guide to understanding the causes, prevention, and treatment of cancer. Massachusetts: Jones \& Bartlett Learning; 1993.

[10] Hewitt M, Weiner SL, Simone JV. Childhood cancer survivorship: Improving care and quality of life. New York: National Academies Press; 2003

[11] Anderson FS, Kunin-Batson AS. Neurocognitive late effects of chemotherapy in children: The past 10 years of research on brain structure and function. Pediatric Blood \& Cancer. 2009; 52(2):159-64. doi: 10.1002/pbc.21700

[12] Ness KK, Armenian SH, Kadan-Lottick N, Gurney JG. Adverse effects of treatment in childhood acute lymphoblastic leukemia: General overview and implications for long-term cardiac health. Expert Review of Hematology. 2011; 4(2):185-97. doi: 10.1586/ehm.11.8

[13] Huang IC, Brinkman TM, Cheung YT, Pui CH, Hudson MM, Krull KR. Functional consequence of cognitive impairment in survivors of childhood Acute Lymphoblastic Leukemia (ALL): the role of cancer symptoms as mediators. Paper presented at: The 2016 American Society of Clinical Oncology (ASCO) Cancer Survivorship Symposium; 2016 January 15-16; San Francisco, California, USA.

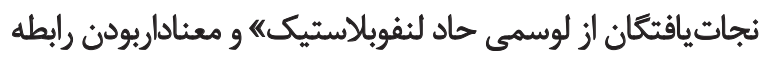

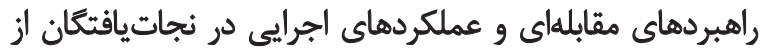

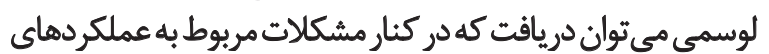

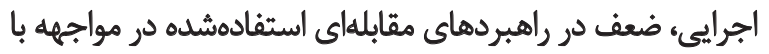

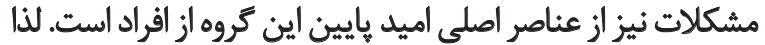

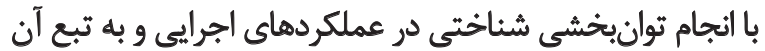

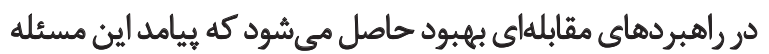
به شكل افزايش نمرههاي اميد خود را نشان مي دهد.

$$
\text { تتيجلمَيرى }
$$

نتايج حاصل از يرؤوهش حاضر نشان داد توانبخشى شئاختى

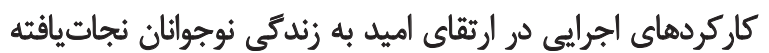

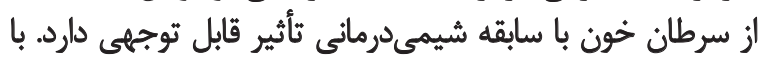

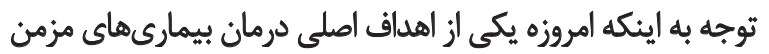

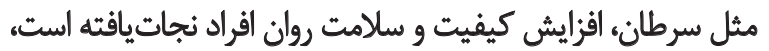

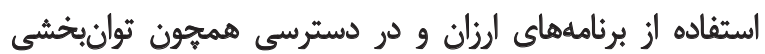

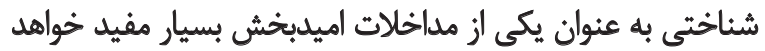

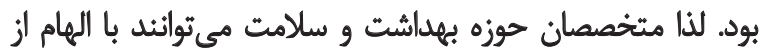

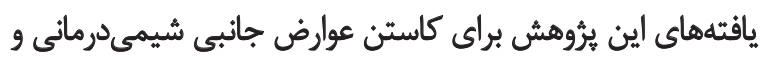

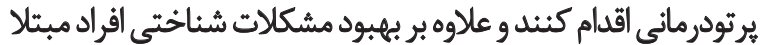
به ارتقاي سطح اميدوارى در اين كروه از بيماران نيز كين كمك كنيند.

ازجمله محدوديتهاى اين يزوهش مى توان به به تعداد اندى

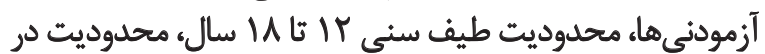

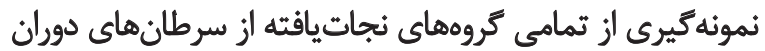

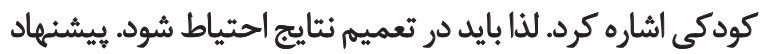

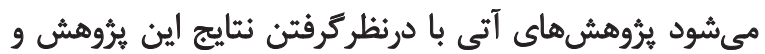

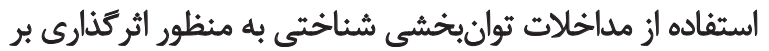

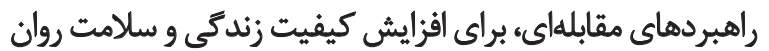

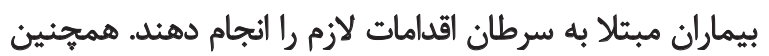

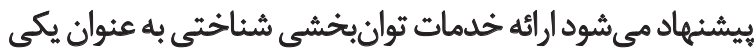

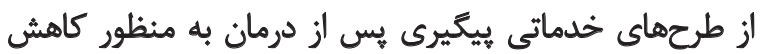

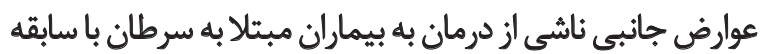
شيمى درمانى در بيمارستان هان ارائه شودان بيمارن

$$
\text { تشكر و قدردانى }
$$

يُروهش حاضر از بايايانامه آقاى اميد امانى در كروه روانشناسى مانى

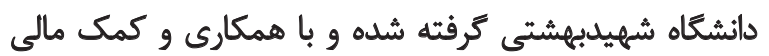

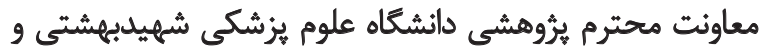

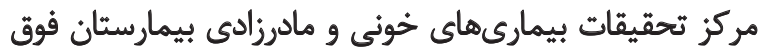

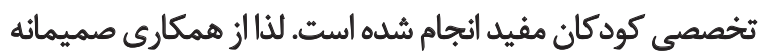

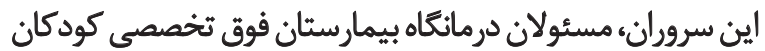

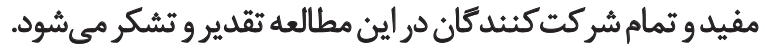


[14] Baker KS, Rajotte EJ. Late effects after treatment for leukemia. leukemia and related disorders. Philadelphia: Springer; 2012.

[15] Walsh KS, Paltin I, Gioia GA, Isquith P, Kadan-Lottick NS, Neglia JP, et al. Everyday executive function in standard-risk acute lymphoblastic leukemia survivors. Child Neuropsychology. 2014; 21(1):78-89. doi: 10.1080/09297049.2013.876491

[16] Abdollahipour F, Alizadeh Zarei M, Akbar Fahimi M, Karamali Esmaeili S. [Study of face and content validity of the Persian version of behavior rating inventory of executive function, preschool version (Persian)]. Journal of Rehabilitation. 2016; 17(1):12-19.

[17] Rabbitt P. Methodology of frontal and executive function. Hove: Psychology Press; 2004.

[18] Shiri V, Hosseini SA, Pishyareh E, Nejati V, Biglarian A. Study the relationship of executive functions with behavioral symptoms in children with high-functioning autism (Persian)]. Journal of Rehabilitation. 2015; 16(3):208-17.

[19] Kavianpour F, Malekpour M, Abedi A. Efficacy of executive functions training (response inhibition) on the rate of impulsivity in preschool children with developmental coordination disorder: a single-subject research (Persian)]. Journal of Rehabilitation. 2013; 14(1):70-80.

[20] Campbell LK, Scaduto M, Van Slyke D, Niarhos F, Whitlock JA, Compas BE. Executive function, coping, and behavior in survivors of childhood acute lymphocytic leukemia. Journal of Pediatric Psychology. 2008; 34(3):317-27. doi: 10.1093/jpepsy/jsn080

[21] Peterson CC, Johnson CE, Ramirez LY, Huestis S, Pai ALH, Demaree HA, et al. A meta-analysis of the neuropsychological sequelae of chemotherapy-only treatment for pediatric acute lymphoblastic leukemia. Pediatric Blood \& Cancer. 2008; 51(1):99-104. doi: 10.1002/pbc.21544

[22] Ashford J, Schoffstall C, Reddick WE, Leone C, Laningham $\mathrm{FH}$, Glass JO, et al. Attention and working memory abilities in children treated for acute lymphoblastic leukemia. Cancer. 2010; 116(19):4638-45. doi: 10.1002/cncr.25343

[23] Buizer AI, de Sonneville LMJ, Veerman AJP. Effects of chemotherapy on neurocognitive function in children with acute lymphoblastic leukemia: A critical review of the literature. Pediatric Blood \& Cancer. 2009; 52(4):447-54. doi: 10.1002/pbc.21869

[24] Dietrich J, Monje M, Wefel J, Meyers C. Clinical patterns and biological correlates of cognitive dysfunction associated with cancer therapy. Oncologist. 2008; 13(12):1285-95. doi: 10.1634/ theoncologist.2008-0130

[25] Cherrier MM, Anderson K, David D, Higano CS, Gray H, Church A, et al. A randomized trial of cognitive rehabilitation in cancer survivors. Life Sciences. 2013; 93(17):617-22. doi: 10.1016/j.lfs.2013.08.011

[26] Schuurs A, Green HJ. A feasibility study of group cognitive rehabilitation for cancer survivors: Enhancing cognitive function and quality of life. Psycho-Oncology. 2012; 22(5):1043-49. doi: 10.1002/pon.3102

[27] Vallat C, Azouvi P, Hardisson H, Meffert R, Tessier C, PradatDiehl P. Rehabilitation of verbal working memory after left hemisphere stroke. Brain Injury. 2005; 19(13):1157-64. doi: 10.1080/02699050500110595
[28] Kesler SR, Lacayo NJ, Jo B. A pilot study of an online cognitive rehabilitation program for executive function skills in children with cancer-related brain injury. Brain Injury. 2010; 25(1):101-12. doi: $10.3109 / 02699052.2010 .536194$

[29] Butler RW. Neurocognitive interventions for children and adolescents surviving cancer. Journal of Pediatric Psychology. 2005; 30(1):65-78. doi: 10.1093/jpepsy/jsi017.

[30] Hardy KK, Willard VW, Bonner MJ. Computerized cognitive training in survivors of childhood cancer a pilot study. Journal of Pediatric Oncology Nursing. 2011; 28(1):27-33.

[31] Patel SK, Katz ER, Richardson R, Rimmer M, Kilian S. Cognitive and problem solving training in children with cancer: A pilot project. Journal of Pediatric Hematology/Oncology. 2009; 31(9):670-7. doi: 10.1097/mph.0b013e3181b25a1d

[32] Hardy KK, Willard VW, Allen TM, Bonner MJ. Working memory training in survivors of pediatric cancer: A randomized pilot study. Psycho-Oncology. 2012; 22(8):1856-65. doi: 10.1002/ pon. 3222

[33] Nejati V, Shahidi Sh, Maleki Gh, Darvishi Lord M. [Cognitive correlates of hope; Evidence from neuropsychological tests (Persian)]. Advances in Cognitive Science. 2013; 14(4):29-38.

[34] Snyder CR, Harris C, Anderson JR, Holleran SA, et al. The will and the ways: Development and validation of an individual-differences measure of hope. Journal of Personality and Social Psychology. 1991; 60(4):570-85. doi: 10.1037/0022-3514.60.4.570

[35] Rustøen T. Hope and quality of life, two central issues for cancer patients: A theoretical analysis. Cancer Nursing. 1995; 18(5):355361. doi: 10.1097/00002820-199510000-00004

[36] Baljani E, Arax M, Mohammad Alizadeh S. [Evaluation of hope in patients with cancer in hospital of Shahid Gazie's Tabrize (Persian)]. Journal of Nursing and Midwifery. 2012; 21(75):1-8.

[37] Buizer AI, de Sonneville LMJ, van den Heuvel-Eibrink MM, Veerman AJP. Chemotherapy and attentional dysfunction in survivors of childhood acute lymphoblastic leukemia: Effect of treatment intensity. Pediatric Blood \& Cancer. 2005; 45(3):28190. doi: 10.1002/pbc.20397

[38] Stam H, Grootenhuis MA, Last BF. The course of life of survivors of childhood cancer. Psycho-Oncology. 2005; 14(3):227-38. doi: $10.1002 /$ pon.839

[39] Marnat GG. Psychological Evaluation Guidelines for clinica psychologists, counselors and psychiatrists [H. Pasha sharifi., M. R. Nikkho Persian trans.] Tehran: Roshd; 2000.

[40] Minaei A. [Adaptation and standardization of child behavior checklist, youth self-report, and teacher's report forms (Persian)]. Research On Exceptional Children. 2006; 6(1):529-558.

[41] Gholami $\mathrm{m}$ pg, sodani $\mathrm{m}$. [The efficacy of group logotherapy on life expectancy and general health education female patients with thalassemia (Persian)]. Knowledge \& Research in Applied Psychology. 2009; 11(42):23-42.

[42] Nejati V, Pouretemad HR, Bahrami H. Attention training in rehabilitation of children with developmental stuttering. Neurorehabilitation. 2013; 32(2):297-303. doi: 10.3233/NRE-130847 
[43] Von Ah D, Russell KM, Storniolo AM, Carpenter JS. Cognitive dysfunction and its relationship to quality of life in breast cancer survivors. Oncology Nursing Forum; 2009; 36(3):326-336. doi: 10.1188/09.ONF.326-334

[44] Nejati V. [Evaluation of relationship between executive function of brain and mental health in older adults (Persian)]. Psychological Studies. 2009; 5(2):27-44.

[45] Snyder CR. The past and possible futures of hope. Journal of Social and Clinical Psychology. 2000; 19(1):11-28. doi: 10.1521/ jscp.2000.19.1.11

[46] Davidson CL, Wingate LR, Slish ML, Rasmussen KA. The great black hope: Hope andits relation to suicide risk among African Americans. Suicide and Life-Threatening Behavior. 2010; 40(2):170-80. doi: 10.1521/suli.2010.40.2.170

[47] Ghodraty-Jabloo V, Alibhai SM, Breunis H, Puts MT. Keep your mind off negative things: Coping with long-term effects of Acute Myeloid Leukemia (AML). Supportive Care in Cancer. 2015; 24(5):2035-45. doi: 10.1007/s00520-015-3002-4 